\title{
Virtual grand rounds in the Calgary Emergency Medicine Department
}

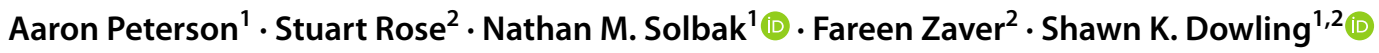 \\ Received: 6 October 2020 / Accepted: 16 October 2020 / Published online: 4 January 2021 \\ (c) Canadian Association of Emergency Physicians (CAEP)/ Association Canadienne de Médecine d'Urgence (ACMU) 2020, corrected publication 2021
}

Keywords Medical education · Virtual education · Web-based learning

In-person grand rounds and continuing medical education (CME) offerings have traditionally been part of academic medical training programs. Due to hospital wide policy regarding physical distancing and the COVID-19 pandemic, on March 19, 2020, we transitioned our emergency department grand rounds from in-person to a virtual format. The purpose of this letter is to describe the successful transition of a Canadian emergency department from in-person to virtual academic departmental rounds and share our learnings.

Advances in virtual meeting technology have allowed communication during COVID-19-enforced isolation. Emergency residency programs have presented live conferences, with $84 \%$ of participants feeling the quality of sessions is the same or better as in-person conference experiences [1]. To keep staff updated with rapidly changing policies and enforce necessary practice changes, Emergency Departments (ED) have presented daily staff updates and biweekly webinars [2].

The initial virtual rounds were a 90-min COVID-19 update to address the changes to our current practice. In subsequent weeks, we returned to the normal pre-pandemic academic rounds schedule of 60-min grand rounds and an additional 45-min ED COVID-19 update session. Iterative improvements have been implemented to address issues encountered. These modifications include: (1) having panelists meet on Zoom 30 min prior to the start of the rounds to check the audio and visual performance and to provide an overview of the virtual rounds format; (2) compiling all presenter slides into a single-slide deck presented by one

Shawn K. Dowling

shawn.dowling@ucalgary.ca

1 Physician Learning Program, Continuing Medical Education and Professional Development, Health Sciences Centre, G-302, Cumming School of Medicine, University of Calgary, 3330 Hospital Drive NW, Calgary, AB T2N 4N1, Canada

2 Department of Emergency Medicine, Cumming School of Medicine, University of Calgary, Calgary, Canada facilitator; (3) sharing the webinar link via email only to prevent unwanted attendees from joining; (4) having one team member with technical experience with the platform to troubleshoot issues. Future plans include increasing the interactivity of the sessions using polling functions, strategies to increase social connectedness, and inviting non-local experts to present at rounds virtually.

The virtual rounds' attendance has fluctuated between 143 and 353 attendees with an average attendance of 211 over nine sessions. Virtual rounds pose unique challenges, but the feedback from staff has been overwhelmingly positive. The COVID-19 pandemic has forced medical teams to innovate their means of updating colleagues on pertinent information and staying connected. Transitioning ED grand rounds to a completely online format represents an opportunity to increase engagement, convenience, and knowledge transfer to physicians. Virtual grand rounds might be a "new normal" that medical departments need to be able to utilize. Shared findings and challenges can help with implementation and ease transition to a virtual format.

Acknowledgments We would like to acknowledge the other members of the COVID virtual grand rounds working group; Dr. Eddy Lang, Dr. Neil Collins, Dr. Andrea Boone, Dr. Gord Mcneil, Dr. Bryan Weber, Dr. Kathryn Crowder, Dr. Jason Fedwick, Dr. Katherine Bateman and Dr. Chris Bond.

Funding This research received no specific grant from any funding agency, commercial, or not-for-profit sectors.

\section{References}

1. Rose C, Mott S, Al'ai Alvarez M. Physically distant, educationally connected: interactive conferencing in the era of COVID-19. Med Educ. 2020;54(8):758-9.

2. Baugh JJ, Sonis JD, Wittbold KA, White BA, Raja AS, Aaronson EL, et al. Keeping pace: an ED communications strategy for COVID-19. Am J Emerg Med. 2020. https://doi.org/10.1016/j.ajem.2020.04.033. 